\title{
Aharonov-Casher phase and persistent current in a polyacetylene ring
}

\author{
Zhijian $\mathrm{Li}^{1}$,J.-Q. Liang ${ }^{1,2}$, F.-C. Pu ${ }^{2}$ \\ ${ }^{1}$ Department of Physics and Institute of Theoretical Physics, \\ Shanxi University, Taiyuan, Shanxi 030006, People's Republic of China \\ ${ }^{2}$ Institute of Physics and center for Condensed Matter Physics, \\ Chinese Academy of Sciences, Beijing 100080, People's Republic of China \\ and Department of Physics, Guangzhou Normal College, \\ Guangzhou 510400, People's Republic of China
}

We investigate a polyacetylene ring in an axially symmetric, static electric field with a modified SSH Hamiltonian of a polyacetylene chain. An effective gauge potential of the single electron Hamiltonian due to spin-field interaction is obtained and it results in a Fröhlich's type of superconductivity equivalent to the effect of travelling lattice wave. The total energy as well as the persistent current density are shown to be a periodic function of the flux of the gauge field embraced by the polyacetylene ring.

Much attention in the study of mesoscopic systems has concentrated upon topological effects in multiply connected geometries. As is well known, the Aharonov-Bohm (AB) effect has led to a number of remarkable interference phenomena in mesoscopic systems, especially in a ring [1]. A typical example of the phenomena first observed by Büttiker et al. is the oscillation of the persistent current in mesoscopic rings threaded by a magnetic flux [1] [2] which leads to a relative phase on the wave function of a charged particle due to the U(1) gauge theory. Since the realization of the Berry phase characterized by a geometric meaning, it has been predicted that analogous interference effects can be induced by the geometric phases which originate from the interplay between an electron's spin and orbital degrees of freedom. Loss et al. studied the persistent current in a one-dimensional ring in the presence of a static inhomogeneous magnetic field considering the coupling between spin 
and orbital motion by the Zeeman interaction [3]. Using the imaginary time path-integral method, they showed that the spin wave function accumulates a Berry phase which leads to a persistent equilibrium current [3]. The Aharonov-Casher(AC) effect in mesoscopic systems has also been of great interest, since it specifically includes the spin degree of freedom. Meir et al. showed that spin orbit interaction(SO) in one dimensional rings results in an effective magnetic flux [4]. Mathur and Stone then pointed out that observable phenomena induced by SO interactions are essentially the manifestation of the AC effect and proposed an experiment to observe the AC oscillation of the conductance in semiconductor samples [5]. Subsequently Balatsky, Altshuler [6] and Choi [7] studied the persistent current related to the $\mathrm{AC}$ effect. Inspired by these studies for textured rings, the $\mathrm{AC}$ effect has also been analyzed in connection with the spin geometric phase. Aronov and Lyanda-Geller considered the spin evolution in conduction rings, and found that SO interaction results in a spin-orbit Berry phase which plays an interesting role in the transmission probability [\$]. However, in realistic systems, the interactions between electrons, lateral dimension, impurities and the coupling of spins complicate the situation. Cheung et al. have systematically studied the persistent currents in ideal, one-dimensional metallic rings, including temperature and impurity effects [9]. Experimental observations of persistent currents have been also reported in an ensemble of ${ }^{\sim} 10^{7} \mathrm{Cu}$ rings [2], in single gold rings [10] and in a single loop in a GaAs heterojunction [11].

It has long been known that the interaction between electrons and the field of lattice displacements may be responsible for superconductivity, known as Fröhlich superconductivity [12]. The average electron momentum corresponds to that of a travelling lattice wave which results in the persistent current in a one-dimensional lattice with periodic boundary condition i.e. a ring. The periodic lattice displacement gives rise to an energy gap in the single-electron spectrum with all levels below the gap occupied and all above it empty. Fröhlich suggested that if, when the electrons was displaced in $k$ space (see Fig.1) so as to give a current flow, the gaps follow the displaced Fermi surface, superconductive behavior might result and is called the fluctuation superconductivity or paraconductivity. The 
existence of the energy gap eliminates the possibility of elastic scattering, provided, the velocity of traveling lattice wave is sufficiently small. Therefore at low temperature an electric current can exist without resistance. Originally, Fröhlich's mechanism of superconductivity because of restriction to one dimension was regarded as a mathematical model without real physical significance. However, Bardeen pointed out that [13 Fröhlich's one-dimensional model rather than a BCS type of pairing was able to account for the origin of superconductivity in the experiment of Coleman et al. [14] who observed an extraordinary increase in conductivity just above the Peierls soft mode instability in a one-dimensional organic solid. Fröhlich's theory originally was based on a nearly-free electron model and applied only to $\mathrm{T}=0 \mathrm{~K}$. This concept i.e. the superconductivity in one-dimensional systems resulted from a coupling between the electrons and a traveling macroscopically occupied lattice wave was further developed by Allender, Bray and Bardeen [15]. In a superfluid there must be macroscopic occupation of a quantum state that picks out a unique reference frame describing the velocity $v_{s}$ of the superfluid [15]. Associated with each value of $v_{s}$ there is a whole set of elementary excitations of the system. When excitations come into equilibrium with the rest frame, a current $j_{s}\left(v_{s}\right)$ remains. In superfluid helium the macroscopic occupation is the Bose condensate of the momentum state and it is the common momentum of pairs that defines $v_{s}$ in a superconductor based on pairing. In the Fröhlich model $v_{s}$ is determined by the velocity of the macroscopically occupied lattice wave which produces energy gaps at boundaries of the displaced Fermi surface 15 (see Fig.1 (b)). It was reported in ref. 16 that the macroscopic velocity $v_{s}$ can be induced by the AB magnetic flux in a polyacetylene ring, where the traveling lattice wave is pinned down due to dimerization and the persistent current is solely due to the AB phase which leads to a collective shift of the electron momentum equivalent to that of traveling lattice wave. Motivated by the formal duality between $\mathrm{AB}$ and $\mathrm{AC}$ effects (but with very different physical origin), we in this paper investigate the AC phase which,we will see, also induces the persistent current in a polyacetylene ring.

Polyacetylene is the simplest linear conjugated polymer. The thermodynamically stable trans configuration is sketched in Fig.2 illustrating the $\sigma$ bonding and the $\pi$ bonding in the 
$\mathrm{x}-\mathrm{y}$ plane. A tight-binding model is suitable for describing the $\pi$ electrons in a polyacetylene chain. A full theory has been given by Su, Schrieffer and Heeger (SSH) [17]. It is certainly interesting to investigate the Fröhlich-like superconductivity related to the AC effect in the context of the modified SSH model. We consider a one-dimensional polyacetylene ring of $\mathrm{N}$ electrons in which there is one conduction electron ( $\pi$ electron) per site with spacing $d$. A infinitely long line charge is assumed to be set along the axis of the ring i.e. the $\mathrm{z}$ axis shown in Fig.3. The AC setup consists of the spin of $\pi$ electron and the electric field of the line-charge. The Lagrangian for the $\pi$ electron with arbitrary polarization of spin can be written in nonrelativistic limit as

$$
L=\frac{m}{2} \dot{\vec{x}}^{2}+\frac{\mu}{c} \dot{\vec{x}} \cdot(\vec{S} \times \vec{E})-\sum_{n=1}^{N} v\left(x-R_{n}\right)
$$

where $\mathrm{m}$ and $\vec{x}$ are the mass and position of the electron, respectively. $\vec{E}=E_{R} \hat{e}_{r}$ represents the radial static electric field of the line-charge with $\mathrm{E}_{R}=\frac{2 \rho}{R}$ where $\rho$ denotes the charge per unit length on the line and $\mathrm{R}$ is the radius of the polyacetylene ring. $\mu=g \mu_{B}$ with $\mu_{B}=$ $\frac{e \hbar}{2 m c}$ being the Bohr magneton. $g$ is the spin $g$ factor which is taken to be 2 here. $\vec{S}$ is then the dimensionless spin operator. $v\left(x-R_{n}\right)$ denotes the potential created by the nth ion with coordinate $R_{n}$. The physical significance of the second term in the Lagragian eq.(1) is clear, since a moving magnetic moment $\mu \vec{S}$ is equivalent to an electric dipole moment $\frac{\mu}{c} \dot{\vec{x}} \times \vec{S}$. Since we consider a one-dimensional ring, the trivial potential energy of charged particle in the electric field has no contribution to the following investigation. The Hamiltonian is

$$
\mathrm{h}=\frac{1}{2 m}\left(\vec{P}-\frac{\mu}{c} \vec{S} \times \vec{E}\right)^{2}+\sum_{n=1}^{N} v\left(x-R_{n}\right)
$$

where $\vec{P}$ is momentum, For the $\pi$ electron confining on the ring, the Hamiltonian eq.(2) is obtained in a cylindrical coordinate as

$$
h=\frac{1}{2 m}\left(-i \hbar \frac{1}{R} \frac{d}{d \varphi}-\frac{\mu}{c} E_{R} S_{z}\right)^{2}+\left(\frac{\mu}{c} E_{R}\right)^{2}(\vec{n} \cdot \vec{S})^{2}+\sum_{n=1}^{N} v\left(x-R_{n}\right)
$$

where

$$
\vec{n}=(\sin \varphi,-\cos \varphi, 0)
$$


is an unit vector showing in Fig.3. The azimuthal angle $\varphi$ is related to the coordinate $\mathrm{x}$ obviously by $x=R \varphi$. Considering a tight-binding model with Wannier function $\phi_{l}$ per site $l$ such that

$$
\left[\frac{\vec{P}^{2}}{2 m}+\nu\left(x-R_{l}\right)\right] \phi_{l}=\varepsilon \phi_{l}
$$

we construct the wave function

$$
\psi_{s}(x)=\xi_{s} \otimes \psi(x)
$$

which is the product of the spinor

$$
\xi_{s}=\frac{1}{\sqrt{2}}(|\vec{n}\rangle+|-\vec{n}\rangle)
$$

and spacial wave function

$$
\psi(x)=\sum_{l=1}^{N} c_{l} \phi_{l}(x)
$$

$| \pm \vec{n}\rangle$ denote the spin coherent states defined by $(\vec{S} \cdot \vec{n})| \pm \vec{n}\rangle= \pm \frac{1}{2}| \pm \vec{n}\rangle$, Taking into account of the unit vector $\vec{n}$ expressed in eq.(4), we have the spin coherent states written explicitly as

$$
\begin{aligned}
|\vec{n}\rangle & =\frac{\sqrt{2}}{2}\left(e^{-\frac{1}{2} i \varphi}|+\rangle+e^{\frac{1}{2} i \varphi}|-\rangle\right) \\
|-\vec{n}\rangle & =\frac{\sqrt{2}}{2}\left(e^{-\frac{1}{2} i \varphi}|+\rangle-e^{\frac{1}{2} i \varphi}|-\rangle\right)
\end{aligned}
$$

where $| \pm\rangle$ are the usual spin eigenstates of $S_{z}, S_{z}| \pm\rangle= \pm \frac{1}{2}| \pm\rangle$.

Averaging on the spin state $\xi_{s}$, we obtain the effective Hamiltonian as

$$
h_{e f f}=\frac{1}{2 m}\left(\vec{P}+\frac{e}{c} \vec{A}\right)^{2}+\sum_{n=1}^{N} v\left(x-R_{n}\right)
$$

where $\vec{A} \equiv \frac{c}{2 e}\left(\hbar \frac{1}{R}+\frac{\mu}{c} E_{R}\right) \hat{\mathrm{e}}_{\varphi}$ is formally equivalent to the vector potential of a magnetic flux with respect to a charged particle with charge e as shown in ref. [16]. $\hat{\mathrm{e}}_{\varphi}$ denotes the unit vector of azimuthal direction. The Hamiltonian operator eq.(10) is formally the same as 
that in ref. [16] where the $\pi$ electron is in the gauge field of a magnetic flux. We therefore confirm the duality between $\mathrm{AB}$ and $\mathrm{AC}$ effects by our model study. Following ref. [16] we assume that $\psi(x)$ is the eigenfunction of $h_{e f f}$, such that

$$
h_{e f f} \psi(x)=E(k) \psi(x)
$$

and satisfies the usual periodic boundary condition

$$
\psi(x+N d)=\psi(x)
$$

Using an unitary transformation

$$
\begin{aligned}
\psi^{\prime}(x) & =\exp \left[i \frac{1}{2 \hbar}\left(\hbar \frac{1}{R}+\frac{\mu}{c} E_{R}\right) x\right] \psi(x) \\
& =\exp \left[i \frac{\Phi_{A C}}{N d \Phi_{0}} 2 \pi x\right] \psi(x)
\end{aligned}
$$

the transformed effective Hamiltonian becomes

$$
\mathrm{h}_{e f f}^{\prime}=\frac{\vec{P}^{2}}{2 m}+\sum_{n=1}^{N} v\left(x-R_{n}\right)
$$

where

$$
\Phi_{A C}=\oint \vec{A} \cdot d \vec{l}=\frac{c}{2 e} N d\left(\hbar \frac{1}{R}+\frac{\mu}{c} E_{R}\right)
$$

is the effective flux of the gauge potential embraced by the ring which we may call the AC flux. $\Phi_{0}=\frac{c h}{e}$ is the quantum unit of the flux for a single electron. The stationary schrödinger equation is seen to be

$$
\mathrm{h}_{\text {eff }}^{\prime} \psi^{\prime}=E(k) \psi^{\prime}
$$

The energy eigenvalue $E(k)$ and the eigenfunction $\psi(x)$ are determined by both eq.(11) and the usual periodic boundary condition eq.(12). Correspondingly $\psi^{\prime}(x)$ possess a nontrivial boundary condition

$$
\psi^{\prime}(x+N d)=\exp \left[i 2 \pi \frac{\Phi_{A C}}{\Phi_{0}}\right] \psi^{\prime}(x)
$$


The vector potential is eliminated in the Hamiltonian $\mathrm{h}_{\text {eff }}^{\prime}$ and the energy spectrum $\mathrm{E}(\mathrm{k})$ is then determined by eq.(16) along with the nontrivial boundary condition of wavefunction eq.(17). The reason why we make use of the unitary transformation eq.(13) to calculate the energy spectrum is that we can therefore adopt Dirac's arguments 18 to demonstrate the periodicity of spectrum with respect to the magnetic flux following Byers and Yang [19]. The situation is exactly the same as that in the analysis of the magnetic flux quantization in superconductivity. Under the unitary transformation the kinetic momentum in the Hamiltonian $h_{\text {eff }}$ eq.(10)

$$
\vec{P}_{k i n}=\vec{P}+\frac{e}{c} \vec{A}
$$

becomes

$$
\vec{P}_{k i n}^{\prime}=\vec{P}
$$

in the Hamiltonian $h_{\text {eff }}^{\prime}$ eq.(14) where $\vec{P}$ denotes the canonical momentum.

Following the argument of Byers and Yang in their famous paper [19] explaining the magnetic flux quantization in superconductor, we immediately conclude that the eigenvalue $E(k)$ should be a periodic function of the $\mathrm{AC}$ flux $\Phi_{A C}$ and an even function of $\Phi_{A C}$. When $\frac{\Phi_{A C}}{\Phi_{0}}$ equals an integer, the boundary condition eq.(17) coincides with the usual one i.e. eq.(12), and then the charged line would not result in any observable effect similar to the $\mathrm{AB}$ case shown in ref. [16] where the magnetic flux becomes a Dirac string. Using eq.(15) it is easy to find that the charge per unit length for the line-charge resulting in no observable effect equals to

$$
\rho=\left(n+\frac{1}{2}\right) \frac{m c^{2}}{e}
$$

where $n$ is an integer. The AC partner of the quantum unit of magnetic flux $\Phi_{0}$ in ref. 16] i.e. in the $\mathrm{AB}$ case is seen to be $\rho_{0}=\frac{m c^{2}}{e}$.

To see how the eigenvalue $\mathrm{E}(\mathrm{k})$ depends on the $\mathrm{AC}$ flux, we evaluate the single-electron energy band with a small variation of AC flux from the integral units of $\Phi_{0}$. The secondquantization Hamiltonian of the electron can be written as 


$$
\begin{aligned}
H= & \int \psi^{+}(x) h_{e f f} \psi(x) d x=\int \psi^{\prime}(x) h_{e f f}^{\prime} \psi^{\prime}(x) d x \\
= & -\sum_{l}\left[t_{0}-\alpha\left(u_{l+1}-u_{l}\right)\right]\left(a_{l+1}^{+} a_{l}+a_{l}^{+} a_{l+1}\right) \\
& +i M v_{f} \sum_{l}\left(a_{l+1}^{+} a_{l}-a_{l}^{+} a_{l+1}\right) \\
& +\frac{1}{2} m v_{f}^{2} \sum_{l} a_{l}^{+} a_{l}
\end{aligned}
$$

The first sum is the usual result in the tight-binding approximation and is seen to be SSH Hamiltonian for electrons. $t_{0}$ denotes the hopping integral in the ion equilibrium position, namely the undimerized chain, while $\alpha$ is the electron-phonon coupling constant. The second and the third sum come from the contribution of the AC flux, where $M \equiv i \int \varphi_{l-1}^{*} \vec{P} \varphi_{l} d x$ is the dipole matrix element which is assumed to be the same for all $l$ and $v_{f}=\frac{2 \hbar \pi}{m N d} \frac{\Phi_{A C}}{\Phi_{0}}$ is regarded as a macroscopic velocity of electrons induced by AC flux i.e.

$$
v_{s}=v_{f}
$$

For the perfectly dimerized chain, the displacement configuration of the lattice may be written as $u_{n}=(-1)^{n} u$. Following SSH we separate the $a_{n}$ into those of odd $\left(a_{n_{o}}\right)$ and even $\left(a_{n_{e}}\right)$ indices and introduce the Fourier transforms

$$
\begin{aligned}
& a_{n_{o}}=\frac{1}{\sqrt{N}} \sum_{k}\left(c_{k}^{v}+c_{k}^{c}\right) e^{-i 2 \pi n_{o} d k} \\
& a_{n_{e}}=\frac{1}{\sqrt{N}} \sum_{k}\left(c_{k}^{v}-c_{k}^{c}\right) e^{-i 2 \pi n_{e} d k}
\end{aligned}
$$

where $|k| \leq \frac{1}{4 d}$, the superscripts $v$ and $c$ denote the valence and conduction bands respectively. The Hamiltonian (10) can be expressed in the $k$ representation.

$$
\begin{aligned}
H= & \sum_{k} E_{0}(k)\left(c_{k}^{v+} c_{k}^{v}-c_{k}^{c+} c_{k}^{c}\right)+i \sum_{k} \Delta(k)\left(c_{k}^{v+} c_{k}^{c}-c_{k}^{c+} c_{k}^{v}\right) \\
& +2 v_{f} M \sum_{k} \sin (2 \pi k d)\left(c_{k}^{v+} c_{k}^{c}-c_{k}^{c+} c_{k}^{v}\right) \\
& +\frac{1}{2} m v_{f}^{2} \sum_{k}\left(c_{k}^{v+} c_{k}^{v}+c_{k}^{c+} c_{k}^{c}\right)
\end{aligned}
$$

where 


$$
\begin{gathered}
E_{0}=-2 t_{0} \cos (2 \pi k d) \\
\Delta(k)=4 \alpha u \sin (2 \pi k d)
\end{gathered}
$$

In order to diagonalize the Hamiltonian (20), we make the following Bogoliubov transformation,

$$
\begin{aligned}
& a_{k}^{v}=-i \alpha_{k} c_{k}^{v}+\beta_{k} c_{k}^{c} \\
& a_{k}^{c}=\alpha_{k}^{*} c_{k}^{v}+i \beta_{k} c_{k}^{c}
\end{aligned}
$$

with $\left|\alpha_{k}\right|^{2}+\left|\beta_{k}\right|^{2}=1$. The diagonalized form is

$$
H=-\sum_{k} E(k)\left(a_{k}^{v+} a_{k}^{v}-a_{k}^{c+} a_{k}^{c}\right)+\frac{1}{2} m v_{f}^{2} \sum_{k}\left(a_{k}^{v+} a_{k}^{v}+a_{k}^{c+} a_{k}^{c}\right)
$$

where

$$
\begin{aligned}
E(k) & =\sqrt{E_{0}^{\prime}(k)^{2}+\Delta(k)^{2}} \\
E_{0}^{\prime}(k) & =E_{0}(k)+2 v_{f} M \sin 2 \pi k d
\end{aligned}
$$

A real solution for the transformation coefficient in eq.(22) is seen to be

$$
\begin{aligned}
& \alpha_{k}=\frac{1}{\sqrt{2}} \sqrt{1+\frac{E_{0}^{\prime}(k)}{E(k)}} \\
& \beta_{k}=-\frac{1}{\sqrt{2}} \frac{k}{|k|} \sqrt{1-\frac{E_{0}^{\prime}(k)}{E(k)}}
\end{aligned}
$$

At zero temperature the valence band is occupied while the conduction band is empty. The total energy is obtained as

$$
U=-2 \sum_{k=-1 / 4 d}^{1 / 4 d} \sqrt{E_{0}^{\prime}(k)^{2}+\Delta^{2}(k)}+\frac{1}{2} N m v_{f}^{2}
$$

Under the condition $\frac{v_{f} M}{t_{0}} \ll \frac{2 \alpha u}{t_{0}} \ll 1$, the first term can be approximated up to quadratic dependence on the small variation of AC flux away from an integer value of $\Phi_{0}$. Since the flux $\Phi_{A C}$ whenever becoming an integral multiple of $\Phi_{0}$ will not affect the energy eigenvalue $E(k)$ i.e. $v_{f}$ vanishes, the total energy would be a minimum seeing from eq.(26). The total 
energy of the occupied valence band has infinite number of minima wherever the magnetic flux is an integral multiple of $\Phi_{0}$. The AC flux dependence of the energy is shown in Fig.4.

To obtain the macroscopic momentum shift, namely the displacement of electrons in $k$ space,we first have to find the electron wave function for the single-electron Hamiltonian $h_{e f f}^{\prime}$. Such a wave function which leads to the valence band $E_{v}=-E(k)$ is

$$
\psi^{\prime}(x)=\frac{\exp \left(i \frac{m v_{f}}{\hbar} x\right)}{\sqrt{N}}\left(\alpha_{k} \sum_{n} e^{i 2 \pi k n d} \phi_{n}+i \beta_{k} \sum_{n} e^{i 2 \pi k n d}(-1)^{n} \phi_{n}\right)
$$

The expectation value of kinetic momentum $\left\langle\vec{P}_{k i n}\right\rangle$ can be calculated with the above wave function,

$$
\begin{aligned}
\left\langle\vec{P}_{k i n}\right\rangle & =\left\langle\psi\left|\vec{P}+\frac{e}{c} \vec{A}\right| \psi\right\rangle \\
& =\left\langle\psi^{\prime}|\vec{P}| \psi^{\prime}\right\rangle \\
& =m v_{f}+\frac{2 M E_{0}^{\prime}(k)}{E(k)} \sin (2 \pi k d)
\end{aligned}
$$

where the contribution from the second term is negligible when summed over $k$. The persistent current density due to a small deviation of AC flux from an integral number of $\Phi_{0}$ is seen to be

$$
j=n e v_{f}
$$

where $n=\frac{1}{d}$ is the electron density . It is seen that the current density depends on AC flux periodically and discontinuous at half-integral values of $\Phi_{0}$, which is shown in Fig.5.

In summary, we study a polyacetylene ring in existence of the radial electric field induced by an infinitely long charge line in the one-dimensional tight-binding model with the electronphonon interaction. An effective gauge potential of $\pi$ electron due to spin-field interaction leads to Fröhhich's type of superconductivity equivalent to the effect of travelling lattice wave. The flux of the gauge potential which results in a collective momentum shift of electron leads to a persistent current, which oscillates with respect to the AC flux and may be observed experimentally with a mesoscopic polyacetylene tube. 
[1] Büttiker M, Imry Y, and Landauer R 1983 Phys. Lett. A 96365

[2] Levy L P, Dolan G, Dunsmuir J and Bouchiat H 1990 Phys. Rev. Lett. 642094

[3] Loss D, Goldbart P and Balatsky A V 1990 Phys. Rev. Lett. 651655 ; Loss D and Goldbart P 1992 Phys. Rev. B 4513544

[4] Meir Y, Gefen Y and Entin-Wohlman O 1989 Phys. Rev. Lett. 63798 ; Entin-Wohlman O, Gefen Y, Meir Y and Oreg Y 1992 Phys. Rev. B 4511890

[5] Mathur H and Stone A D 1992 Phys. Rev. Lett. 682964

[6] Balatsky A V and Altshuler B L 1993 Phys. Rev. Lett. 701678

[7] Choi M Y 1993 Phys. Rev. Lett. 712987

[8] Aronov A G and Lyanda-Geller Y B 1993 Phys. Rev. Lett. 70343 ; Lyanda-Geller Y 1993 Phys. Rev. Lett. 71657

[9] Cheung H F, Gefen Y, Riedel E K and Shih W H 1988 Phys. Rev. B 376050

[10] Chandrasekar V,Webb R A, Brady M J, Ketchen M B, Gallagher W J and Kleinsasser A 1991 Phys. Rev. Lett. 673578

[11] Mailly D, Chapelier C and Benoit A 1993 Phys. Rev. Lett. 702020

[12] Fröhlich H 1954 Proc. R. Soc. Ser. A 223296

[13] Bardeen J 1973 Solid State Commun. 13 357; Allender D, Bray J W and Bardeen J 1974 Phys. Rev. B 9119

[14] Coleman L B, Cohen M J, Sandman D J, Yamagishi F G, Garito A F and Heeger A J 1973 Solid State Commun. 121125

[15] Allender D, Bray J W and Bardeen J 1974 Phys. Rev. B 9119

[16] Liang J Q, Peng F and Ding X X 1995 Phys. Lett. A 201369 
[17] Su W P, Schrieffer J R and Heeger A J 1978 Phys. Rev. Lett. 42 1968; Su W P, Schrieffer J R and Heeger A J 1980 Phys. Rev. B 222099

[18] Dirac P A M 1948 Phys. Rev. 74817

[19] Byers N and Yang C N 1961 Phys. Rev. Lett. 746

\section{Figure Caption}

Fig.1 (a) The half-filled tight-binding band which has undergone a Peierls transition. There is no current $\left(v_{s}=0\right)$. (b) Same as (a) except $v_{s} \neq 0$.

Fig.2 Trans configuration of $(\mathrm{CH})_{x}$.

Fig. $3 \pi$ electron with arbitrary polarization of spin $\vec{S}$ in the electric field of charge line along $z$ direction.

Fig.4 The variation of the total energy as a function of AC flux.

Fig.5 Periodic variation of current density as a function of $\mathrm{AC}$ flux. 


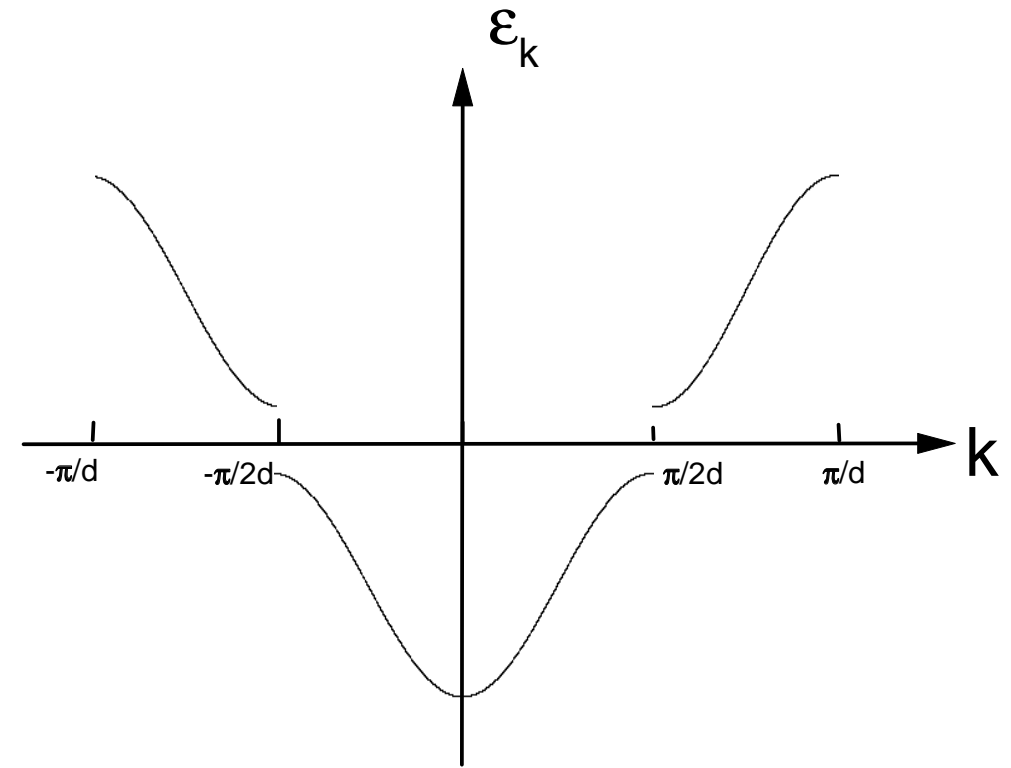

(a)

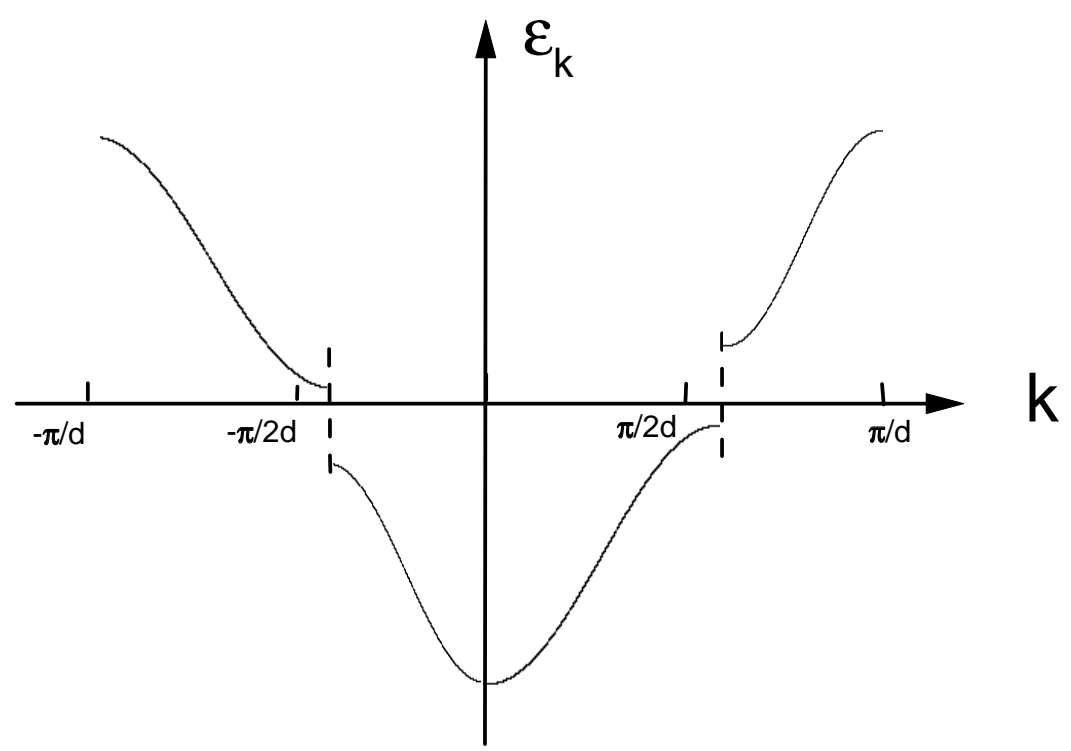

(b)

Fig.(1) 


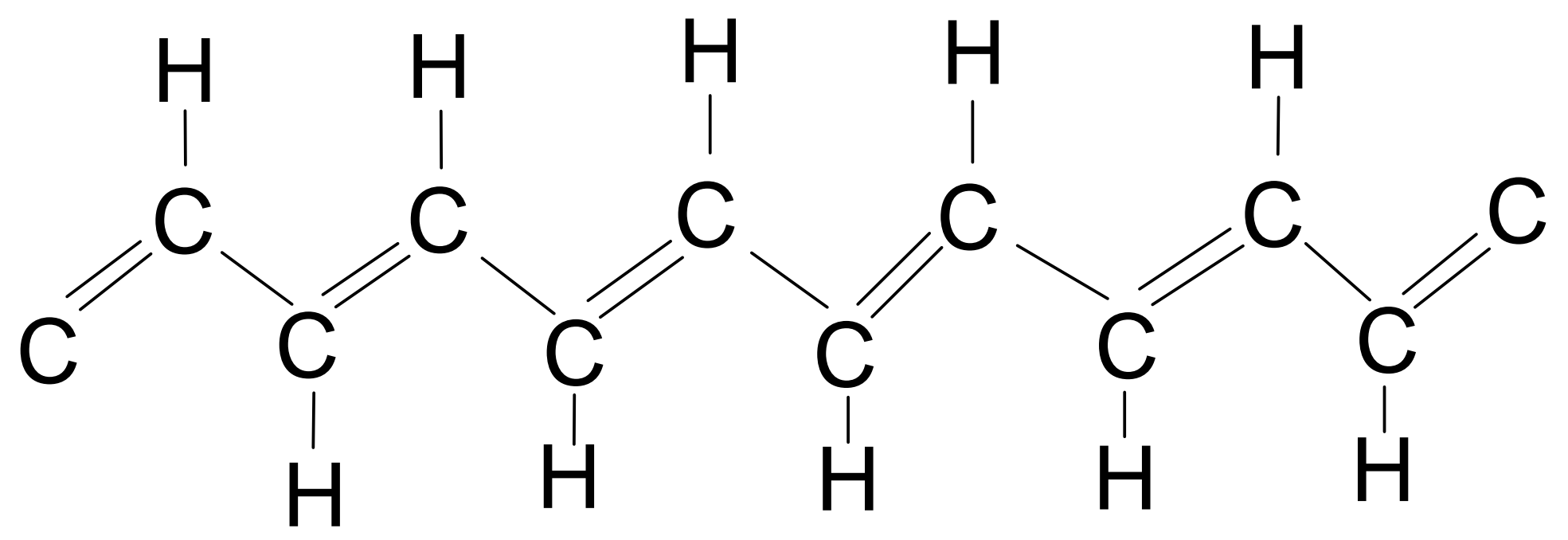

Fig. 2 


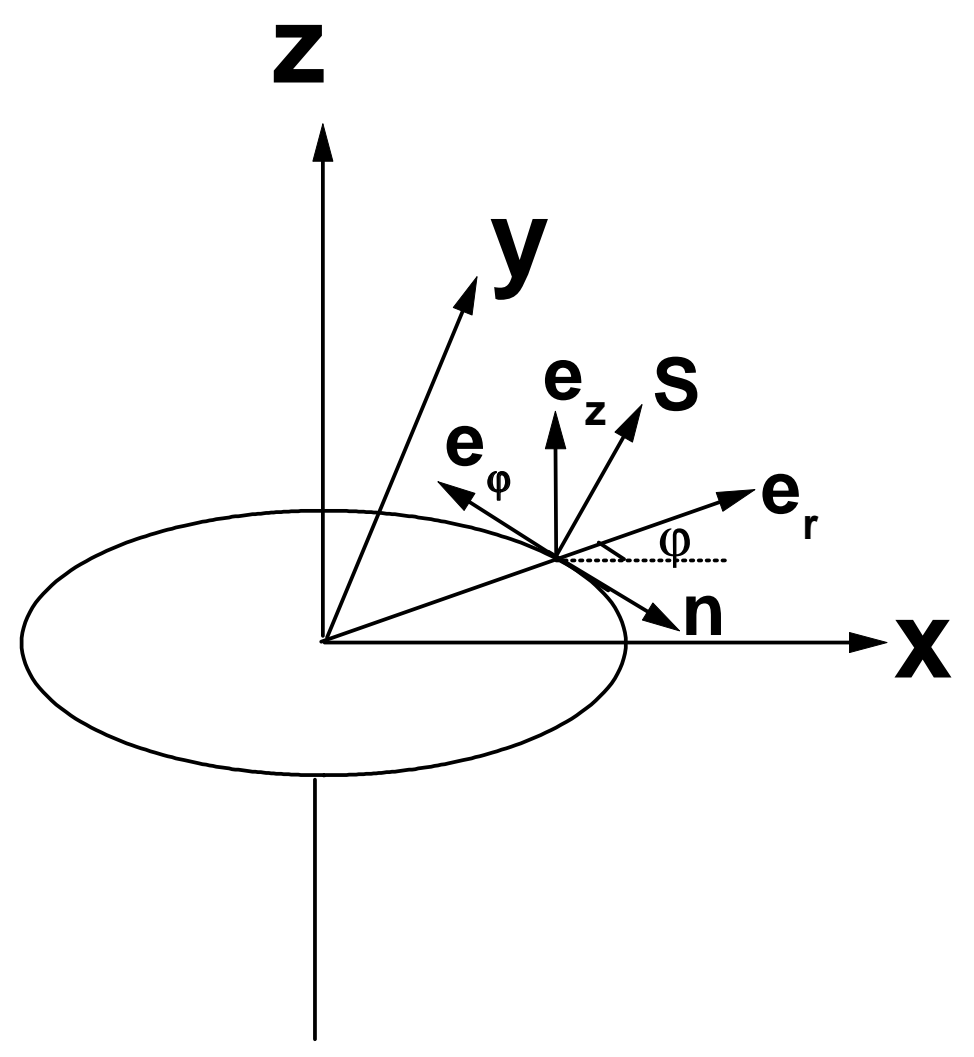

Fig. 3 


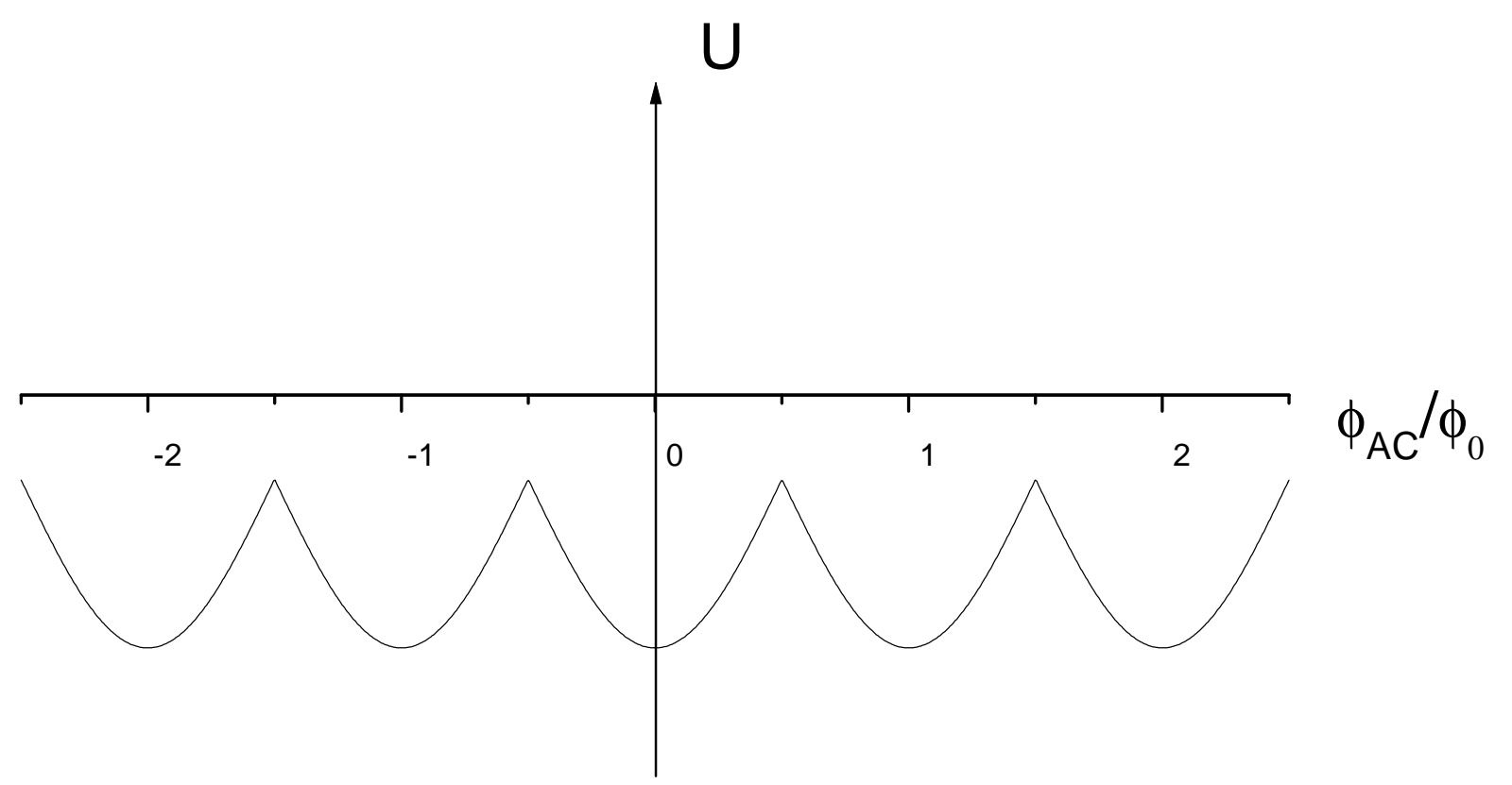

Fig. 4 


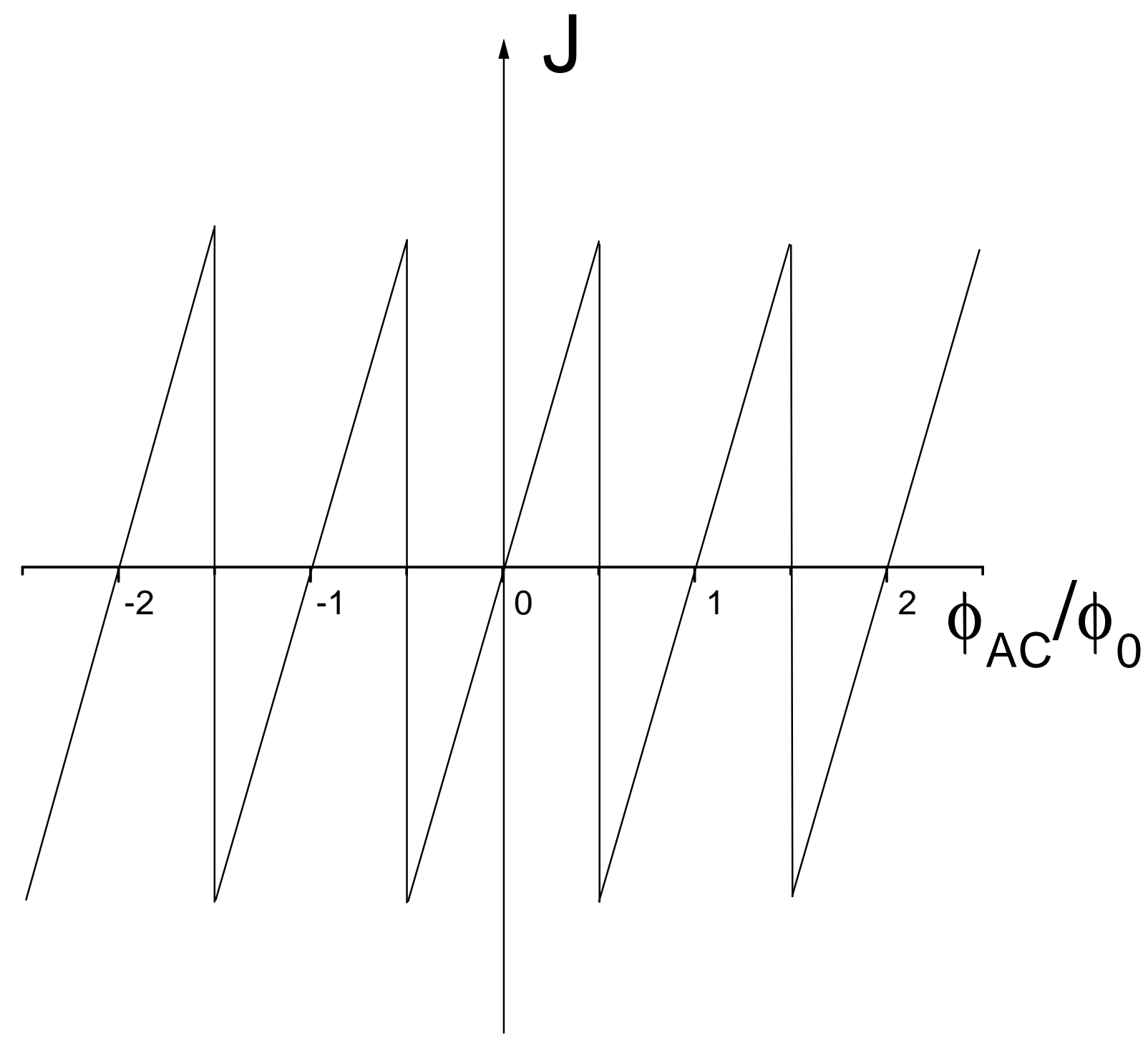

Fig.5 